Clinical Physiology and
Functional Imaging

\title{
Oesophageal Doppler guided optimisation of cardiac output does not increase visceral microvascular blood flow in healthy volunteers
}

\begin{tabular}{|c|c|}
\hline Journal: & Clinical Physiology and Functional Imaging \\
\hline Manuscript ID & CPF-2016-0064.R1 \\
\hline Manuscript Type: & Original Article \\
\hline Date Submitted by the Author: & 28-Jul-2016 \\
\hline Complete List of Authors: & $\begin{array}{l}\text { Heinink, Tom; University of Nottingham, Division of Medical Sciences and } \\
\text { Graduate Entry Medicine } \\
\text { Read, David; University of Nottingham, Division of Medical Sciences and } \\
\text { Graduate Entry Medicine } \\
\text { Mitchell, William; University of Nottingham, Division of Medical Sciences } \\
\text { and Graduate Entry Medicine } \\
\text { Bhalla, Ashish; University of Nottingham, Division of Medical Sciences and } \\
\text { Graduate Entry Medicine } \\
\text { Phillips, Beth; University of Nottingham, Division of Medical Sciences and } \\
\text { Graduate Entry Medicine } \\
\text { Lund, Jon; University of Nottingham, Division of Medical Sciences and } \\
\text { Graduate Entry Medicine } \\
\text { Williams, John; University of Nottingham, Division of Medical Sciences and } \\
\text { Graduate Entry Medicine }\end{array}$ \\
\hline Key Words: & $\begin{array}{l}\text { Contrast-enhanced, Ultrasound, oesophageal, Doppler, healthy volunteers, } \\
\text { cardiac output, visceral, perfusion }\end{array}$ \\
\hline
\end{tabular}




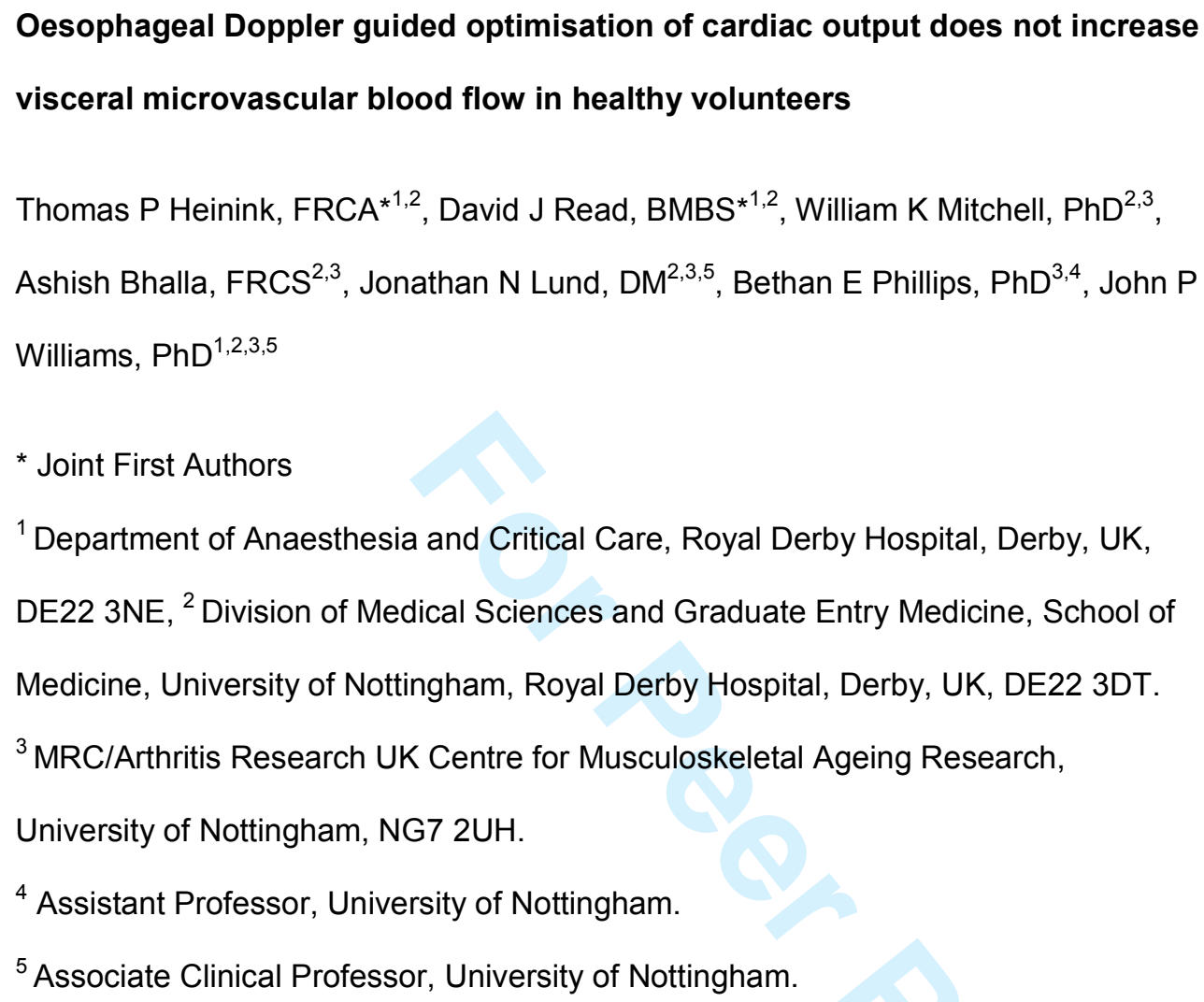

Abbreviated title: Doppler guided cardiac output and microvascular blood flow

\section{Corresponding Author:}

Dr David J Read,

Room 5102, School of Graduate Entry Medicine and Health,

University of Nottingham,

Royal Derby Hospital,

Derby, DE22 3DT.

E-mail: dread1@nhs.net

Telephone: 01332724731 


\section{Contact Details:}

Dr Thomas P Heinink - tomheinink@doctors.org.uk

Mr William K Mitchell - kylemitchell@doctors.org.uk

Mr Ashish Bhalla - ashbhalla@doctors.org.uk

Dr Bethan E Phillips - beth.phillips@nottingham.ac.uk;

Mr Jonathan N Lund - Jon.Lund@nottingham.ac.uk

Dr John P Williams - john.williams7@nhs.net 


\section{Background}

3 Oesophageal Doppler Monitoring (ODM) is used clinically to optimise cardiac output

4 (CO) and guide fluid therapy. Despite limited experimental evidence, it is assumed

5 that increasing $\mathrm{CO}$ increases visceral microvascular blood flow (MBF). We used

6 contrast-enhanced ultrasound (CEUS) to assess if ODM-guided optimisation of CO

7 altered MBF.

\section{Methods}

10 Sixteen healthy male volunteers $(62 \pm 3.4$ years) were studied. Baseline measurements of $\mathrm{CO}$ were recorded via ODM. Hepatic and renal MBF were assessed via CEUS. Saline $0.9 \%$ was administered to optimise CO according to a standard protocol and repeat CEUS performed. Time-intensity curves were constructed, allowing organ perfusion calculation via time to $5 \%$ perfusion (TT5). MBF was assessed via organ perfusion rise time (5-95\%) (RT).

Results

CO increased $(4535 \pm 241 \mathrm{ml} / \mathrm{min}$ vs $5442 \pm 329 \mathrm{ml} / \mathrm{min}, \mathrm{p}<0.0001)$ following fluid administration, while time to renal $(22.48 \pm 1.19$ secs. vs. $20.79 \pm 1.31$ secs; $p=0.03)$, but not hepatic $(28.13 \pm 4.48 \mathrm{~s}$. vs $26.83 \pm 1.53 \mathrm{secs} ; \mathrm{p}=0.15)$ perfusion decreased. Time to renal perfusion was related to $\mathrm{CO}$ (renal: $r=-0.43, p=0.01$ ). Hepatic nor renal 
22 RT altered following fluid administration (renal: $9.03 \pm 0.86$ vs. $8.93 \pm 0.85$ secs

$23 p=0.86$; hepatic: $27.86 \pm 1.6$ vs. $30.71 \pm 2.19$ secs, $p=0.13$ ). No relationship was

24 observed between changes in $\mathrm{CO}$ and MBF in either organ (renal: $r=-0.17, p=0.54$;

25 hepatic: $r=-0.07, p=0.80)$.

\section{Conclusions}

ODM optimised CO reduces time to renal perfusion but does not alter renal or hepatic MBF. A lack of relationship between microvascular visceral perfusion and CO following ODM-guided optimisation may explain the absence of improved clinical 31 outcome with ODM monitoring.

\section{Trial Registration}

The study was registered at clinicaltrials.gov (reference number NCT02167178).

\section{Keywords}

Contrast-enhanced Ultrasound, CEUS, oesophageal Doppler, healthy volunteers, cardiac output. 
1

2

3

4

5

6

7

8

9

\section{Background}

The ability to measure cardiovascular performance is integral to anesthetic and critical care practice. Traditional clinical monitoring modalities such as blood pressure $(\mathrm{BP})$, heart rate $(\mathrm{HR})$, and central venous pressure fail to provide a continuous, accurate assessment of microvascular haemodynamic performance or identify instances of tissue hypoperfusion [1, 2] with uncorrected tissue hypoperfusion increasing surgical morbidity and mortality [3].

Alternative monitoring techniques provide estimates of stroke volume (SV) in an attempt to guide fluid and vasoactive drug therapy and optimise tissue perfusion. Traditional measurement of SV involved insertion of a pulmonary artery flotation catheter (PAFC) and measurement via thermodilution techniques. PAFC use has declined over the past decade, primarily due to concerns about the complications of insertion and an absence of studies demonstrating clinical benefit $[4,5]$. Consequently, less invasive techniques for measuring SV have been developed.

Thermodilution, however, remains the gold standard for the assessment of SV against which new monitors are compared [6]. 
developed to guide intravenous (IV) fluid administration [8-11]. It is recommended for intra-operative use by the National Institute for Health and Care Excellence (NICE) and has been advocated for use in awake patients [12].

SV and cardiac output (CO) are intrinsically linked, with CO the product of SV and HR. Whilst ODM permits reproducible estimates of $\mathrm{CO}$, it is unclear what benefits are brought to the patient by its use. Despite studies initially suggesting a reduction in morbidity and mortality with ODM guided perioperative fluid therapy [13, 14], recent randomised controlled trials and meta-analysis' have questioned these conclusions $[15,16]$. CO monitoring provides more information than pressure-related measures, but it is limited to the assessment of changes in whole-body haemodynamics. The complexity of regulatory mechanisms that have been observed to impact upon blood flow through the abdominal organs would suggest that no simple relationship can exist between $\mathrm{CO}$ and visceral perfusion. This challenges the notion that clinical benefit will directly result from maximisation of $\mathrm{CO}$. Therefore, assessment of visceral microvascular blood flow (MBF) (e.g. in the gastrointestinal mucosa during and after abdominal surgery) may provide more relevant end points for guiding fluid therapy to reduce perioperative visceral hypoperfusion.

Contrast enhanced ultrasound (CEUS) is an imaging modality that can provide nearreal time imaging of perfusion within viscera at a capillary level. CEUS has been validated for accurately measuring visceral blood flow against a number of proven technologies. Numerous in-vitro and in vivo studies, have validated the accuracy of 
1

2

3

4

5

6

7

87

88

89

90

91

92

93

94

95

96

97

98

99

100

101

CEUS in assessing microvascular blood flow, demonstrating close correlation with thermodilution [17], mechanically controlled flow [18] and end organ microvascular perfusion [19], [20] .

CEUS utilises echogenic microspheres that return a characteristic echo pattern.

During CEUS, intravenous administration of a bolus of the contrast agent permits construction of time-acoustic intensity (Al) curves. From these curves the time from bolus to $5 \%$ of peak Al (TT5) for each organ, pre- and post-fluid administration and rise time (RT), defined as the time taken to rise from $5-95 \%$ of the peak Al (Figure 1), may be calculated. This technique has previously been validated as a method of tracking changes in MBF of the intra-abdominal viscera [21, 22].

We hypothesised that administration of intravenous (IV) fluid to achieve ODM-guided CO optimisation would reliably track visceral perfusion in both liver and kidney of a healthy individual. 
Methods

103

104
The University of Nottingham Medical School Research Ethics Committee (A12012012) granted ethical approval for the study. The study was registered at clinicaltrials.gov (reference number NCT02167178) and conformed to the Declaration of Helsinki. Sixteen healthy male participants aged between 18 and 80 years were recruited using a standard demographically targeted postal invite.

Participants attended for a pre-study health screening appointment and written informed consent was obtained. Participants were excluded if they presented with:

$\mathrm{BMI}<20$ or $>30 \mathrm{~kg} \mathrm{~m}^{-2}$, recent acute coronary syndrome, use of $\beta$-blockers, cerebrovascular disease, metabolic disease, known malignancy, clotting dysfunction, previous oesophageal surgery or oesophageal varices, history of epistaxis or known sensitivity to SonoVue ${ }^{\mathrm{TM}}$. For subject demographics see Table 1.

\section{Subject preparation}

Subjects attended the University of Nottingham; Clinical, Metabolic and Molecular Physiology laboratories fasted for 12 hours of food and fluids. A medically qualified doctor was present throughout the study and subjects were continuously monitored with pulse oximetry $\left(\mathrm{SpO}_{2}\right)$, electrocardiogram (ECG) and non-invasive blood pressure recording (NIBP). A 20G intravenous cannula was sited in the right antebrachial vein and an $18 \mathrm{G}$ in the left. Venous blood was drawn for measurement of haemoglobin concentration $(\mathrm{Hb})$ and haematocrit $(\mathrm{Hct})$. A trans-oesophageal Doppler probe (Deltex Medical, Chichester, UK) was inserted into the oesophagus via the nostril, following local anesthesia to the naso-pharynx with $10 \%$ lidocaine 
1

2

3

4

5

6

7

8

9
12

spray and $2 \%$ lidocaine gel (ClinMed Ltd, High Wycombe, United Kingdom). The probe was connected to a CardioQ Oesophageal Doppler Monitor (ODM) (Deltex Medical) and probe position was corrected to achieve an optimal Doppler flow signal. ODM placement was well tolerated by all subjects.

\section{Contrast agent}

SonoVue ${ }^{\mathrm{TM}}$ (Bracco SpA, Milan, Italy), an established contrast agent for quantitative CEUS [23] was used, with preparation as per the manufacturer's instruction [24]. In brief, $25 \mathrm{mg}$ of lyophilised powder was reconstituted with $5 \mathrm{ml}$ of $0.9 \%$ sodium chloride solution $(\mathrm{NaCl})$ in an $\mathrm{SF}_{6}$ atmosphere.

\section{Ultrasound settings}

A Philips iU22 ultrasound machine (Philips Healthcare, Reigate, UK) with a C5-1 MHz curvilinear probe (Philips Healthcare) was used for all examinations, using dual contrast/tissue side-by-side mode. Cine recordings were made at $9 \mathrm{~Hz}$ with a contrast resolution of $\mathrm{C} 40$, a working mechanical index (MI) of 0.04 , a maximum depth of $16 \mathrm{~cm}$ and focus at $8-14 \mathrm{~cm}$. Gain was optimised for each subject.

\section{Experimental protocol}

Patients were placed in a semi-recumbent position. The ultrasound probe was positioned to allow concurrent imaging of the liver and right kidney with probe 
position manipulated to optimise visualised liver and renal parenchyma. Following optimisation the probe position was marked with ink to facilitate repeat visceral imaging.

Once the probe was positioned and marked baseline recordings of $\mathrm{SpO}_{2}, \mathrm{ECG}$, mean arterial blood pressure (MAP), HR and SV were made. CEUS was then performed by administering a rapid bolus of $0.5 \mathrm{ml}$ of SonoVue ${ }^{\mathrm{TM}}$ via the $20 \mathrm{G}$ cannula, immediately followed by a rapid flush of $5 \mathrm{ml}$ of $0.9 \% \mathrm{NaCl}$. At the same time, a continuous, real-time low Ml ultrasound recording of the liver and kidney commenced, and continued for 2 minutes. After each 2 minute cycle, a 5 minute pause was observed, to allow elimination of microbubbles. During which time $\mathrm{SpO}_{2}$, MAP, SV and HR were again measured. This sequence was repeated three times.

Subjects were then given a $250 \mathrm{ml}$ bolus of $0.9 \% \mathrm{NaCl}$ solution as rapidly as possible via the $18 \mathrm{G}$ cannula with a $50 \mathrm{ml}$ syringe and 3-way tap used to facilitate rapid infusion of an accurate fluid volume. On completion of this bolus, SV, HR, NIBP and $\mathrm{SpO}_{2}$ were recorded. Repeat fluid boluses were administered and observations made until the SV no longer increased by $>10 \%$, at which point the SV was deemed optimal [11]. Median fluid administration to optimise SV was 1000ml (IQR 1000$1000 \mathrm{ml}$, range $1000-2000 \mathrm{ml})$. Immediately after optimisation of SV a further set of CEUS recordings and cardiovascular observations were performed, using the protocol outlined above. A further blood sample was then taken for measurements of 
1

2

3

4

7

8

9
168 hemoglobin $(\mathrm{Hb})$ and hematocrit (Hct). Patients were monitored for 30 minutes

169 following completion of the study protocol (Figure 2).

Image processing

172 Ultrasound video files were analysed using QLAB ${ }^{\mathrm{TM}}$ software (Philips Healthcare).

173 Regions-of-interest (ROI) were defined within liver and kidney images to allow 174 computation of the mean pixel intensity within each ROI for each frame of the 175 ultrasound loop (Figure 3). The ROI was chosen to ensure as large an area as 176 possible was available for analysis, whilst avoiding tissue close to the capsule of 177 each organ to minimise the effect of the subtle movement of these organs seen with 178 respiration. Large hilar blood vessels were excluded from the ROI to achieve 179 preferential assessment of microvascular haemodynamics.

182 For each bolus injection, ROI Al was calculated for liver and kidney from each frame 183 (i.e. at $9 \mathrm{~Hz}$ ) and subsequently standardised to that organs maximum intensity. second moving average. The resultant time-intensity trace was used to measure RT (time from $5-95 \%$ of peak $\mathrm{Al}$ ) and TT5 (time from bolus to $5 \%$ of peak $\mathrm{Al}$ ) for each organ pre- and post-fluid administration. Results were averaged across the 3 cycles recorded at each time-point. 
Cardiovascular parameter analysis

Data for SV, MAP, $\mathrm{HR}, \mathrm{Hb}$, Hct and $\mathrm{SpO}_{2}$ were recorded as described above and 192 data stored on an Excel spreadsheet (Microsoft Corporation, Redmond, Washington,

3

USA). Mean values for each of these variables before and after SV optimisation were recorded.

195

196 Statistics

197 Sample size calculations required $n=16$ (for $\alpha=0.05, \beta=0.85$ ), to detect a $30 \%$

198 change in hepatic microvascular blood flow, results we have been able to achieve for 199 previous work looking at similar physiological systems. Statistical analysis was

200 performed using GraphPad Prism ${ }^{\text {TM }}$ v6.0 (La Jolla, CA. USA). Distribution of data 201 was tested using Kolmogorov-Smirnov tests, with normal data expressed as mean \pm 202 standard error of the mean (SEM) and non-normal data as median \pm interquartile 203 range. Independent $t$-tests were applied to normal data and Mann-Whitney tests to 204 non-normal data. Categorical values were compared using Fisher's test. $p<0.05$ was 205 considered significant.

206

207

208

209 
$211 \mathrm{CO}$ and $\mathrm{SpO}_{2}$ increased significantly following fluid administration (4535 \pm 241 vs. $2125442 \pm 329 \mathrm{ml} \cdot \mathrm{min}^{-1}, P<0.0001 ; 96.9 \pm 0.4$ vs. $97.8 \pm 0.3 \%, p<0.01$, respectively), whilst

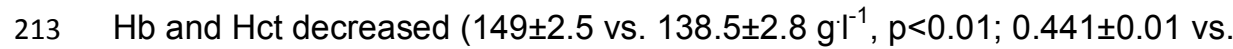
$0.412 \pm 0.01, p<0.01$, respectively). MAP and HR remained unchanged following fluid 215 administration (105.3 \pm 2.4 vs. $106.3 \pm 2.8 \mathrm{mmHg}, \mathrm{p}=0.31 ; 61.8 \pm 1.8$ vs. $62.1 \pm 1.9 \mathrm{bpm}$, $216 p=0.54$, respectively).

217

Despite increases in $\mathrm{CO}$ and decreases in Hct following fluid administration, MBF was not altered by fluid administration in either the hepatic (RT: $27.86 \pm 1.6$ vs. $30.71 \pm 2.19$ secs, $p=0.13$ ) or renal (RT: $9.03 \pm 0.86$ vs. $8.93 \pm 0.85$ secs, $p=0.86$ ) circulation (Figure 4). Likewise no relationship was observed between $\mathrm{CO}$ and MBF in either the kidney $(r=-0.17, p=0.54)$ or liver $(r=-0.07, p=0.8)$ (Figure 5).

Time to renal perfusion decreased following fluid administration (TT5: $22.48 \pm 1.19$ vs. $20.79 \pm 1.31$ secs, $p=0.03$ ), whilst time to hepatic perfusion was unaltered (TT5: $28.13 \pm 4.48$ vs. $26.83 \pm 1.53$ secs, $p=0.15$.). Similarly time to renal, but not hepatic perfusion, was correlated with $\mathrm{CO}$ (renal: $r=-0.43, p=0.01$; hepatic: $r=-0.21, p=0.26$ ) 228 (Figure 5).

There was no significant relationship observed between change in cardiac output ( $\Delta$ $\mathrm{CO})$ and change in renal rise time ( $\Delta$ renal $\mathrm{RT}),(r=-0.17$ and $p=0.27)$. A significant 
232 correlation was observed between $\Delta C O$ and change in renal TT5 ( $\Delta$ TT5), $(r=-0.50$,

$233 p=0.05$; Figure 6).

234

235 In the hepatic circulation, $\Delta \mathrm{CO}$ did not correlate with change in hepatic rise time $(\Delta$ 236 hepatic RT), $(r=0.07, p=0.40)$; nor with change in hepatic TT5 ( $\Delta$ hepatic TT5),

$237 \quad(r=0.09, p=0.36)$.

\section{Discussion}

240 In this study we use the novel technologies of CEUS and ODM to explore the 241 relationship between $\mathrm{CO}$ and MBF. As expected fluid administration reliably 242 increased CO, reduced time to renal perfusion and reduced haematocrit. Despite 243 these changes in macrocirculatory variables, $\mathrm{CO}$ showed no significant correlation 244 with measures of MBF in either renal or hepatic circulations.

245

246 The relationship between venous filling and SV is relatively simple, and is described 247 by the Frank-Starling law; essentially, higher filling pressures lead to greater preload, 248 and hence more forceful contraction of myocardial fibers, resulting in a greater SV 249 and thus CO [25] (other afterload mediated effects remaining constant over the short 250 period of this study). 
8

9

252 The relationship between MBF and fluid administration is more complex, with 253 multiple factors affecting perfusion of the liver and kidney. Strong autoregulatory 254 mechanisms exist within the kidney to maintain a constant blood flow across a range 255 of blood pressures and volaemic conditions [26]. In this healthy volunteer study 256 these mechanisms are likely to have remained intact.

257

The autoregulatory ability of the liver is less robust; with the main determinants of hepatic perfusion being sympathetic nervous system activity, circulating catecholamines, and the interaction between the arterial and portal venous circulations (the hepatic arterial buffer response) [27]. In hypovolaemia, large volumes of blood may be mobilised from the splanchnic circulation to preserve perfusion of the brain, heart and musculature [28]. Hypovolaemia reduces splanchnic perfusion, portal venous flow and hence hepatic blood flow and these effects persist for some time after restoration of euvolaemic [29].

These complex interactions challenge simplistic assumptions that SV and $\mathrm{CO}$ are key determinants of MBF. As microvascular perfusion is vital for normal organ function and tissue healing, including for example, at anastomoses, this lack of response to SV optimisation with intravenous fluid may help to explain why recent publications and meta-analyses have failed to show a consistent reduction in morbidity or mortality when ODM-guided fluid management protocols have been used in the perioperative period $[15,16]$. 
There are a number of limitations to this present study. Firstly, the use of healthy subjects may limit the applicability of the findings to the perioperative and critical care patient. Also in an attempt to somewhat mirror a clinical population subjects were taken from a predominantly older male age range, which may limit the conclusions of this study to a wider clinical group. Subjects were hypovolaemic after a 12-hour fast, as evidenced by the increase in SV with intravenous administration of c. $1 \mathrm{~L}$ of IV crystalloid, and this reflects modern surgical practice [30]. However, the impact of anaesthesia has not been addressed in this study. Additionally, as ODM measurement of cardiac output varies with change in subject position, it was decided that subjects should studied in a semi-recumbent position to aid subject comfort. This position corresponds to the recommended positioning for patients on the intensive care unit. Importantly participant position was not altered between CEUS measurements, in order to reduce any error due to change in subject or probe positioning. However, findings may therefore not be relevant in a population in a fully recumbent position.

The absolute values of $\mathrm{CO}$ measured in this study by ODM are in several instances around $3 \mathrm{~L}$ per minute. This is lower than would be expected for a healthy male population and may relate to position and relatively increased age of the study volunteers. In addition, although ODM measurements were taken by clinicians, experienced and skilled in the use of ODM monitoring, there are undoubted limitations to the use of ODM to acquire exact discrete measures of cardiac output. Furthermore, ODM calculates the volume of blood transiting the descending aorta and employs a number of assumptions to calculate cardiac output from this, while by necessity excluding perfusion of head and upper limbs. Although these factors may 
have resulted in lower than expected numerical values for $\mathrm{CO}$ the ability of the ODM to accurately determine changes in cardiac output is preserved.

Efforts were made to ensure consistency of tissue imaged throughout. Despite this, absolute probe fixation is not possible and small movements, such as with respiration, induce movement artifact to CEUS measures [31]. To overcome this problem, we employed a validated time-based surrogate for tissue perfusion, the RT, which is more robust to small variations in the imaged tissue [22]. This technique does provide a less comprehensive assessment of microvascular status than techniques that generate volumetric data [21, 31], such as microbubble destructionreplenishment [18], but is ultimately more reliable in this cohort of subjects.

A sample size calculation was determined for the primary hypothesis of a $30 \%$ change in hepatic microvascular blood flow following fluid optimisation. Despite ODM assessed fluid optimisation we found no significant change in hepatic microvascular blood flow. Of note, the study was not powered to expose a relationship between the change in $\mathrm{CO}$ and change in MVBF before and after fluid optimization and thus may have been underpowered for detect such a relationship. It is important however to note, that there was also no suggestion of a clear relationship between CO and RT ( $r=-0.07$ (hepatic), $r=-0.17$ (renal)).

\section{Conclusion}

This study describes a bolus method for comparison of ODM-derived CO and CEUSderived measures of renal and hepatic perfusion in the healthy, awake subject. Our data suggest that ODM guided fluid administration reliably increases $\mathrm{CO}$ and time to 
6

322 renal perfusion, but that such changes do not increase MBF within hepatic or renal

323 parenchyma. This challenges the assumption that optimisation of CO improves

324 abdominal visceral perfusion. The inability of ODM-guided fluid management to 325 increase renal and hepatic MBF may be a factor in the lack of improved clinical 326 outcome with ODM monitoring. 


\title{
Competing Interests
}

The other authors declare that they have no competing interests.

\author{
Author contributions \\ $\mathrm{TPH}$ - Analysis and acquisition of data, drafting/revising article, final approval of \\ submitted article \\ DJR - Data analysis, drafting/revising article, final approval of submitted article \\ WKM - Concept and design of study, data acquisition and analysis, drafting/revising \\ article, final approval of submitted article \\ $A B$ - Concept and design of study, data acquisition and analysis, revising article, \\ final approval of submitted article
}


BEP - Concept and design of study, data acquisition and analysis, revising article, final approval of submitted article

JNL - Concept and design of study, data analysis, revising article, final approval of submitted article

JPW - Concept and design of study, data analysis, revising article, final approval of submitted article

\section{Acknowledgements}

The authors would like to thank Mrs Amanda Gates and Mrs Margaret Baker for their assistance with volunteer recruitment and data acquisition.

This work was funded by a grant from the Bowel Disease Research Foundation. 


\section{References}

1. Wo CC, Shoemaker WC, Appel PL, Bishop MH, Kram HB, Hardin E: Unreliability of blood pressure and heart rate to evaluate cardiac output in emergency resuscitation and critical illness. Crit Care Med 1993, 21(2):218-223.

2. Hamilton-Davies C, Mythen MG, Salmon J, Jacobson D, Shukla A, Webb A: Comparison of commonly used clinical indicators of hypovolaemia with gastrointestinal tonometry. Intensive Care Med 1997, 23(3):276-281.

3. Mythen MG, Webb AR: Intra-operative gut mucosal hypoperfusion is associated with increased post-operative complications and cost. Intensive Care Med 1994, 20(2):99-104.

4. Harvey S, Harrison DA, Singer M, Ashcroft J, Jones CM, Elbourne D, Brampton W, Williams D, Young D, Rowan K: Assessment of the clinical effectiveness of pulmonary artery catheters in management of patients in intensive care (PAC-Man): a randomised controlled trial. Lancet 2005, 366(9484):472-477.

5. Sandham JD, Hull RD, Brant RF, Knox L, Pineo GF, Doig CJ, Laporta DP, Viner S, Passerini L, Devitt H: A randomized, controlled trial of the use of pulmonary-artery catheters in high-risk surgical patients. N Engl J Med 2003, 348(1):5-14.

6. Lee AJ, Cohn JH, Ranasinghe JS: Cardiac output assessed by invasive and minimally invasive techniques. Anesthesiology research and practice 2011, 2011.

7. Dark PM, Singer M: The validity of trans-esophageal Doppler ultrasonography as a measure of cardiac output in critically ill adults. Intensive Care Med 2004, 30(11):2060-2066.

8. Conway D, Mayall R, Abdul - Latif M, Gilligan S, Tackaberry C: Randomised controlled trial investigating the influence of intravenous fluid titration using oesophageal Doppler monitoring during bowel surgery. Anaesthesia 2002, 57(9):845-849.

9. Wakeling H, McFall M, Jenkins C, Woods W, Miles W, Barclay G, Fleming S: Intraoperative oesophageal Doppler guided fluid management shortens postoperative hospital stay after major bowel surgery. Br J Anaesth 2005, 95(5):634-642.

10. Noblett S, Snowden C, Shenton B, Horgan A: Randomized clinical trial assessing the effect of Doppler-optimized fluid management on outcome after elective colorectal resection. Br J Surg 2006, 93(9):10691076.

11. Lowe G, Chamberlain B, Philpot E, Willshire R: Oesophageal Doppler Monitor (ODM) guided individualised goal directed fluid management (iGDFM) in surgery - a technical review. In. Edited by Deltex Medical: Deltex Medical; 2010.

12. Medical technologies guidance MTG3: CardioQ-ODM oesophageal doppler monitor. In.: National Institute for Health and Clinical Excellence; 2011.

13. Walsh S, Tang T, Bass S, Gaunt M: Doppler - guided intra - operative fluid management during major abdominal surgery: systematic review and meta - analysis. Int J Clin Pract 2008, 62(3):466-470. 
14. Abbas S, Hill A: Systematic review of the literature for the use of oesophageal Doppler monitor for fluid replacement in major abdominal surgery. Anaesthesia 2008, 63(1):44-51.

15. Srinivasa S, Lemanu DP, Singh PP, Taylor MH, Hill AG: Systematic review and meta-analysis of oesophageal Doppler-guided fluid management in colorectal surgery. Br J Surg 2013, 100(13):1701-1708.

16. McKenny M, Conroy P, Wong A, Farren M, Gleeson N, Walsh C, O'Malley C, Dowd N: A randomised prospective trial of intra-operative oesophageal Doppler-guided fluid administration in major gynaecological surgery. Anaesthesia 2013, 68(12):1224-1231.

17. Herold IH, Russo G, Mischi M, Houthuizen P, Saidov T, van het Veer M, van Assen $\mathrm{HC}$, Korsten $\mathrm{HH}$ : Volume quantification by contrast-enhanced ultrasound: an in-vitro comparison with true volumes and thermodilution. Cardiovasc Ultrasound 2013, 11(1):36.

18. Wei K, Jayaweera AR, Firoozan S, Linka A, Skyba DM, Kaul S: Quantification of myocardial blood flow with ultrasound-induced destruction of microbubbles administered as a constant venous infusion. Circulation 1998, 97(5):473-483.

19. Wei K, Le E, Bin J-P, Coggins M, Thorpe J, Kaul S: Quantification of renal blood flow with contrast-enhanced ultrasound. J Am Coll Cardiol 2001, 37(4):1135-1140.

20. Rim SJ, Leong-Poi H, Lindner JR, Couture D, Ellegala D, Mason H, Durieux M, Kassel NF, Kaul S: Quantification of cerebral perfusion with "RealTime" contrast-enhanced ultrasound. Circulation 2001, 104(21):2582-2587.

21. Gauthier TP, Averkiou MA, Leen EL: Perfusion quantification using dynamic contrast-enhanced ultrasound: the impact of dynamic range and gain on time-intensity curves. Ultrasonics 2011, 51(1):102-106.

22. Gauthier TP, Wasan HS, Muhammad A, Owen DR, Leen EL: Assessment of global liver blood flow with quantitative dynamic contrast-enhanced ultrasound. J Ultrasound Med 2011, 30(3):379-385.

23. Mitchell WK, Phillips BE, Williams JP, Rankin D, Smith K, Lund JN, Atherton PJ: Development of a new Sonovueilliams JP - enhanced ultrasound approach reveals temporal and age - related features of muscle microvascular responses to feeding. Physiological Reports $201 \overline{1}, 1(5)$.

24. Summary of product characteristics [http://www.medicines.org.uk/emc/medicine/7777/SPC/SonoVue+8+microlitre s+ml,+powder+and+solvent+for+dispersion+for+injection/\#SHELF LIFE]

25. Patterson S, Starling E: On the mechanical factors which determine the output of the ventricles. The Journal of Physiology 1914, 48(5):357-379.

26. Giebissch G, Windhager E: Control of Renal Blood Flow and Glomerular Filtration. In: Medical Physiology. 2 edn. Edited by Boron W, Boulpaep E. Philadelphia: Saunders; 2012.

27. Takala J: Determinants of splanchnic blood flow. British Journal of Anaesthesia 1996, 77(1):50-58.

28. Gelman S, Mushlin PS: Catecholamine-induced changes in the splanchnic circulation affecting systemic hemodynamics. Anesthesiology 2004, 100(2):434-439.

29. Edouard A, Degrémont A-C, Duranteau J, Pussard E, Berdeaux A, Samii K: Heterogeneous regional vascular responses to simulated transient hypovolemia in man. Intensive Care Med 1994, 20(6):414-420. 
30. Gustafsson UO, Scott MJ, Schwenk W, Demartines N, Roulin D, Francis N, McNaught CE, Macfie J, Liberman AS, Soop M et al: Guidelines for perioperative care in elective colonic surgery: Enhanced Recovery After Surgery (ERAS) Society recommendations. World J Surg 2013, 37(2):259284.

31. Tang MX, Mulvana H, Gauthier T, Lim AK, Cosgrove DO, Eckersley RJ, Stride E: Quantitative contrast-enhanced ultrasound imaging: a review of sources of variability. Interface Focus 2011, 1(4):520-539. 
Tables

\begin{tabular}{|c|c|c|}
\hline A. & Mean & Standard Deviation \\
\hline Age (years) & 62 & \pm 13.6 \\
\hline Height (m) & 1.76 & \pm 0.06 \\
\hline Weight (kg) & 83.8 & \pm 10.3 \\
\hline $\mathrm{BMI}\left(\mathrm{kg} / \mathrm{m}^{2}\right)$ & 27.1 & \pm 2.4 \\
\hline
\end{tabular}

Formatted: Font: Bold, Font color: Text 1 Formatted: Font: Bold, Font color: Text 1 Formatted: Font: Bold, Font color: Text 1

Formatted: Font color: Text 1

Formatted: Font color: Text 1

Formatted: Font color: Text 1

Formatted: Font color: Text 1

Formatted: Font color: Text 1

Formatted: Font: (Default) Arial 


\section{Legends for Figures}

Figure 1. Example time-intensity curve for the liver. Dotted lines show 5 and $95 \%$ of

Formatted: Font: $12 \mathrm{pt}$ the maximum values. In this example, the $5 \%$ value is 0.037 arbitrary units $(A U)$, occurring at 18.41 seconds (TT5). The $95 \%$ value is $0.699 \mathrm{AU}$, occurring at 32.83 seconds, resulting in a rise time of 14.42 seconds.

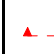

Formatted: Font: $12 \mathrm{pt}$

Figure 2. Outline of study protocol. SV, Stroke Volume; CEUS, Contrast Enhanced Ultrasound; $\mathrm{Hb}$, Haemoglobin; Hct, Haematocrit; $\mathrm{SpO}_{2}$, Oxygen saturation; NIBP, Non-invasive blood pressure; ECG, Electrocardiogram. 1

Formatted: Font: $12 \mathrm{pt}$

Figure 3. Example of region of interest quantification in $\mathrm{QLAB}^{\mathrm{TM}}$ software. Top regions of interest defined on the contrast-enhanced image of the liver (red) and kidney (yellow), Bottom - graph of acoustic intensity against time, as output from $\mathrm{QLAB}^{\mathrm{TM}}$ for liver (red) and kidney (yellow).

Comment [BP1]: Page charges will be higher for colour figures you many want to check this with JW?

Formatted: Font: $12 \mathrm{pt}$

Formatted: Font: $12 \mathrm{pt}$

Figure 4. Normalised Cardiac output, renal rise-time and hepatic rise-time before and after fluid optimisation, ${ }^{* * * *}$ significant difference, pre- vs. post-fluid administration, $p<0.0001$.

Figure 5. Rise time (RT, sec) within the hepatic (A) and renal (B) microcirculations plotted against cardiac output (hepatic $r=-0.07, p=0.8_{\text {i浐 }}$ renal $r=-0.17, p=0.54$ ). Time 
to $5 \%$ perfusion (TT5, sec) within the hepatic (C) and renal (D) microcirculations plotted against cardiac output (hepatic $r=-0.21, p=0.26$, renal $r=-0.43, p=0.01$ ).

Figure 6. Change in rise time $(\Delta \mathrm{RT}$, sec) within the hepatic $(\mathrm{A})$ and renal $(\mathrm{B})$ microcirculation plotted against change in cardiac output $(\Delta \mathrm{CO}, \mathrm{l} / \mathrm{min})$. Change in Time to $5 \%$ perfusion ( $\triangle$ TT5, sec) within the hepatic (C) and renal (D) microcirculations plotted against change in cardiac output $(\Delta C O, I / \min )$,
Formatted: Font: $12 \mathrm{pt}$

Formatted: Font: $12 \mathrm{pt}$, Not Highlight

Formatted: Font: (Default) Arial, $12 \mathrm{pt}$ 
Figure 1. Example time-intensity curve for the liver. Dotted lines show 5 and $95 \%$ of the maximum values. In this example, the $5 \%$ value is 0.037 arbitrary units (AU), occurring at 18.41 seconds (TT5). The $95 \%$ value is $0.699 \mathrm{AU}$, occurring at 32.83 seconds, resulting in a rise time of 14.42 seconds. 
1

2

3

4

5

6

7

8

9

10

11

12

13

14

15

16

17

18

19

20

21

22

23

24

25

26

27

28

29

30

31

32

33

34

35

36

37

38

39

40

41

42

43

44

45

46

47

48

49

50

51

52

53

54

55

56

57

58

59

60

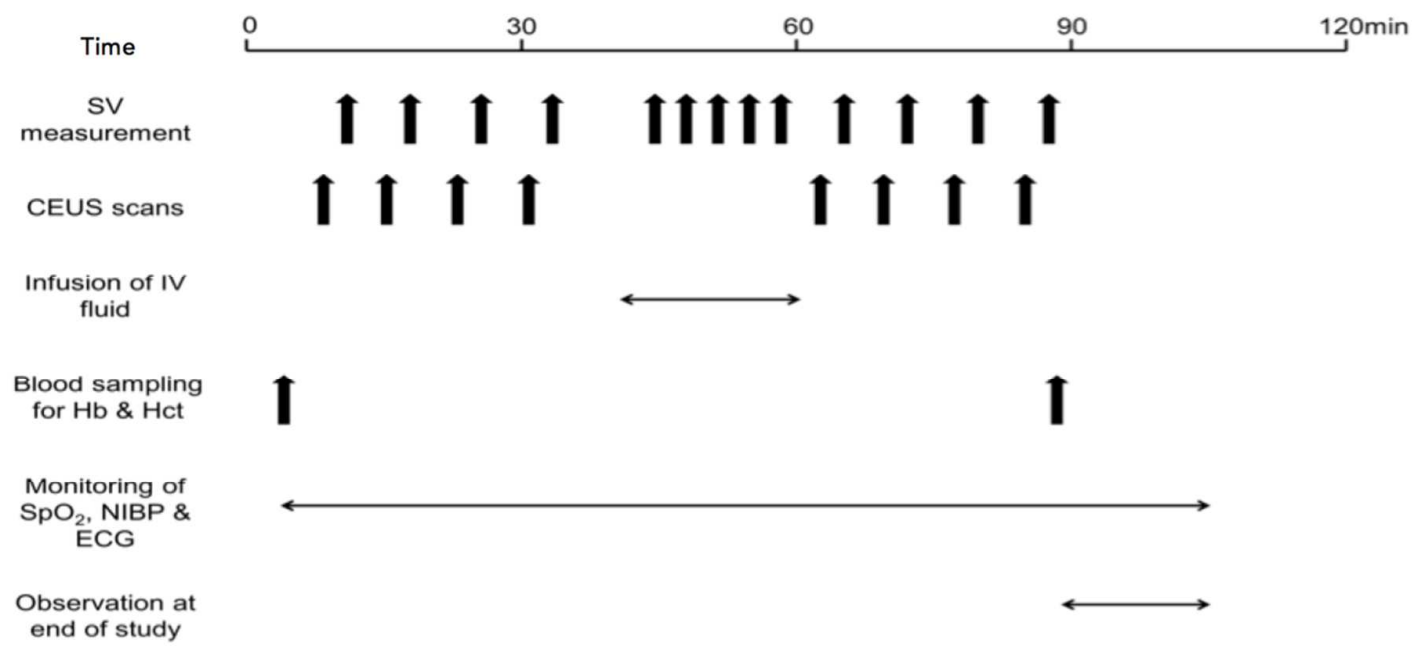

Figure 2 
Figure 3. Example of region of interest quantification in QLAB ${ }^{\mathrm{TM}}$ software. Top - regions of interest defined on the contrast-enhanced image of the liver (red) and kidney (yellow), Bottom - graph of acoustic intensity against time, as output from QLAB ${ }^{\text {TM }}$ for liver (red) and kidney (yellow).
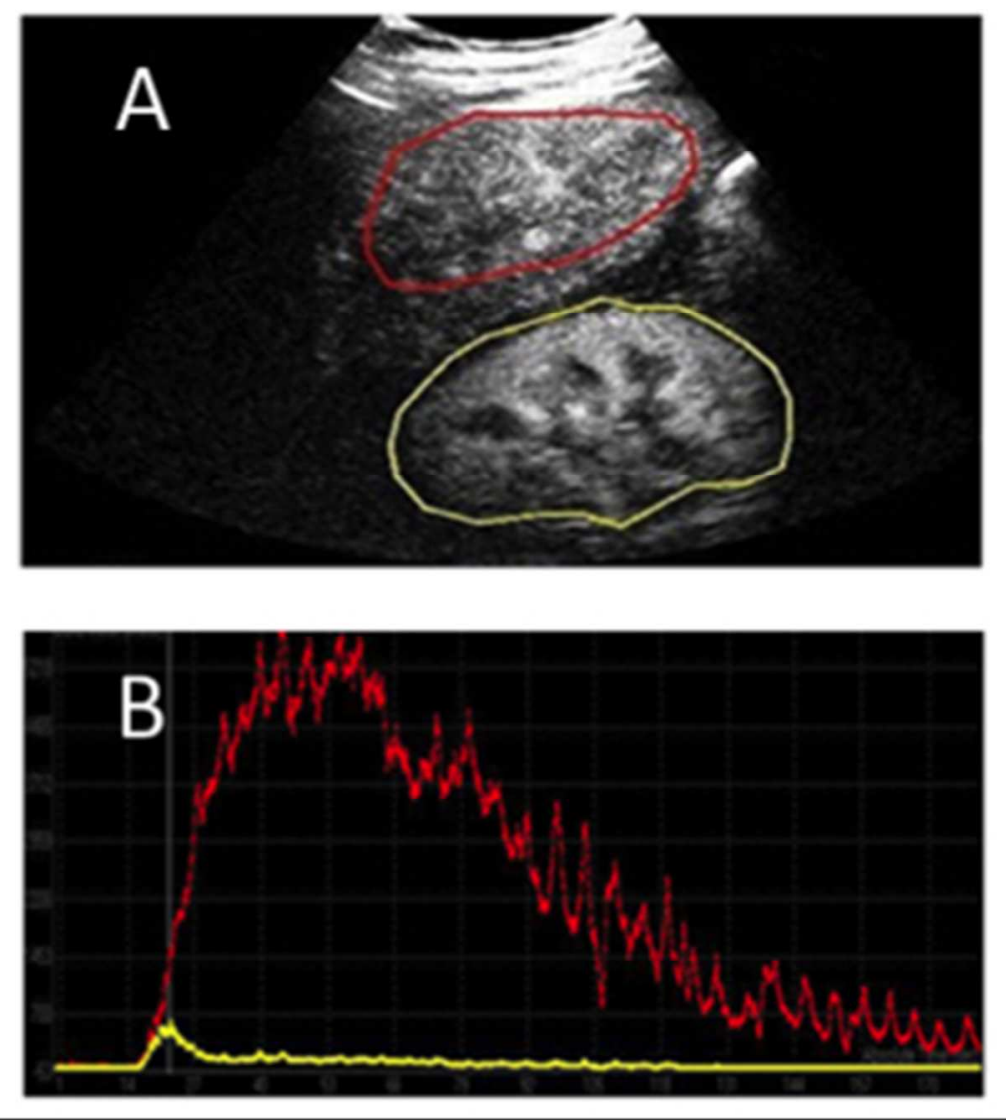

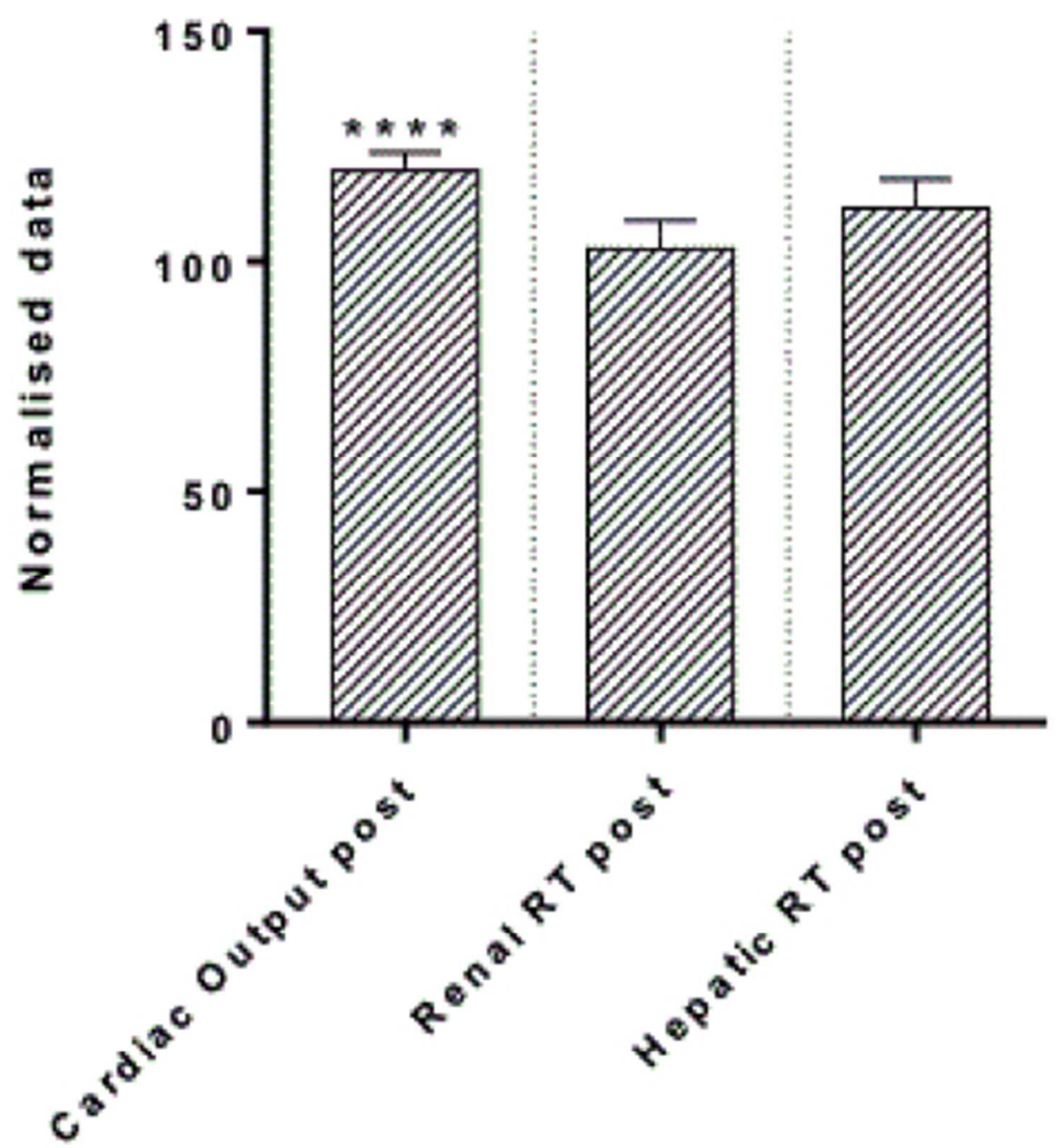

Figure 4. Normalised Cardiac output, renal rise-time and hepatic rise-time before and after fluid optimisation, $* * * *$ significant difference, pre- vs. post-fluid administration, $\mathrm{p}<0.0001$. 


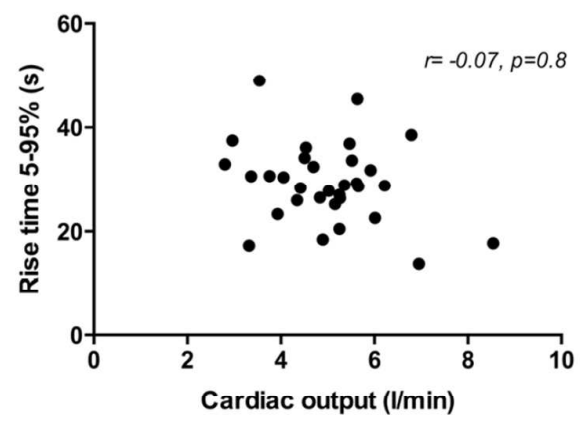

A.
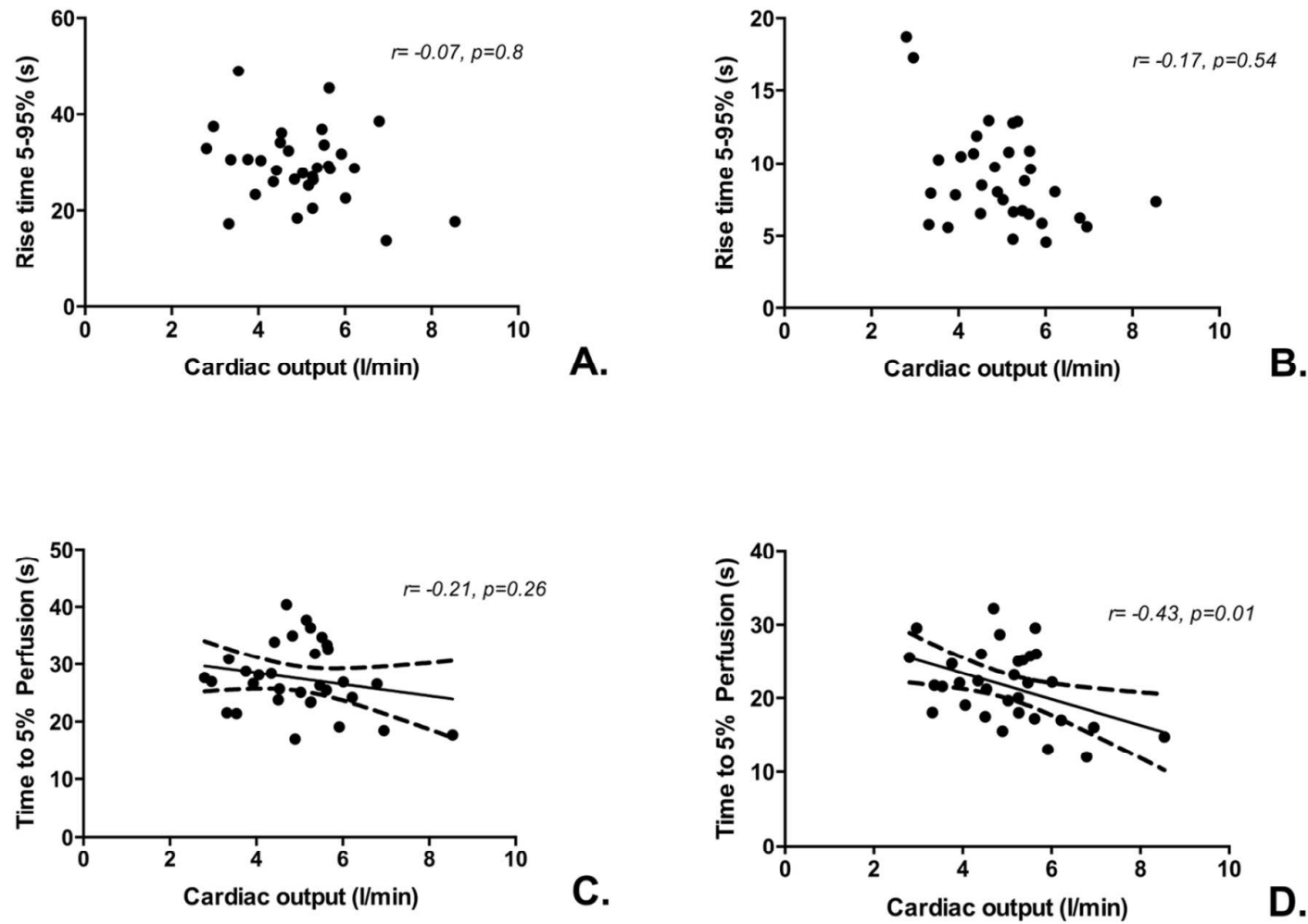

c.

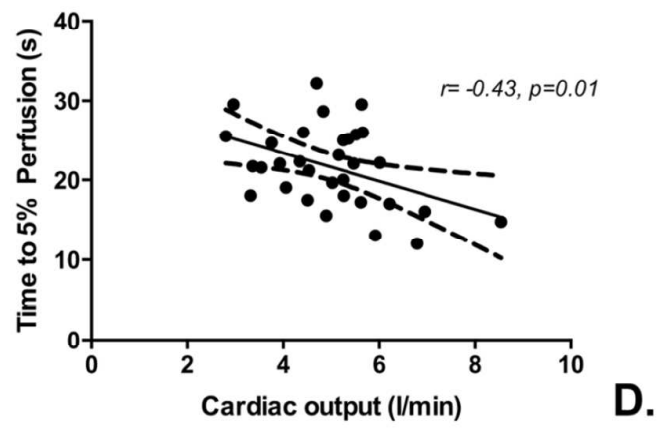

Figure 5 
A

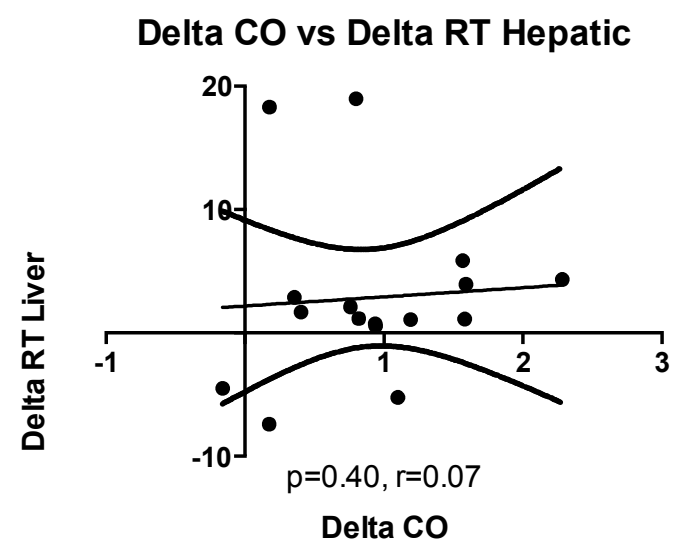

C

Delta CO vs Delta TT5 Hepatic

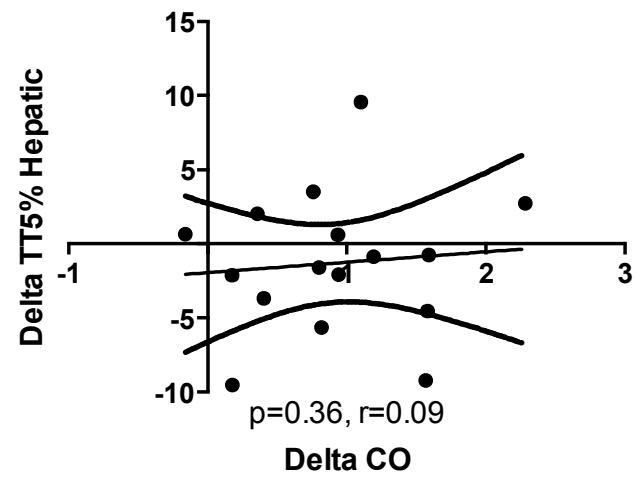

B

Delta CO vs Delta RT Kidney

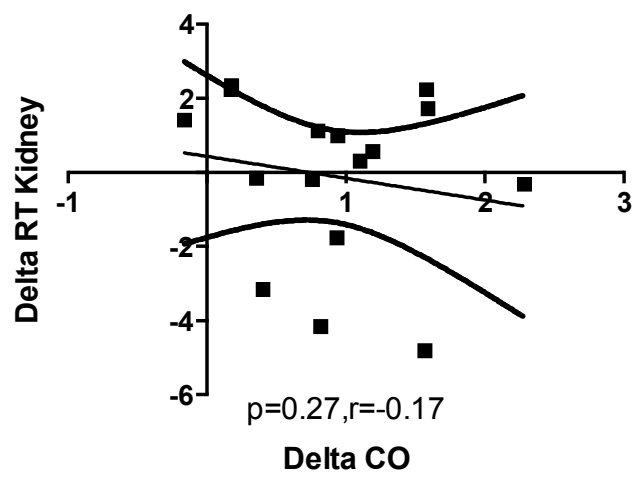

D

Delta CO vs Delta TT5 Kidney

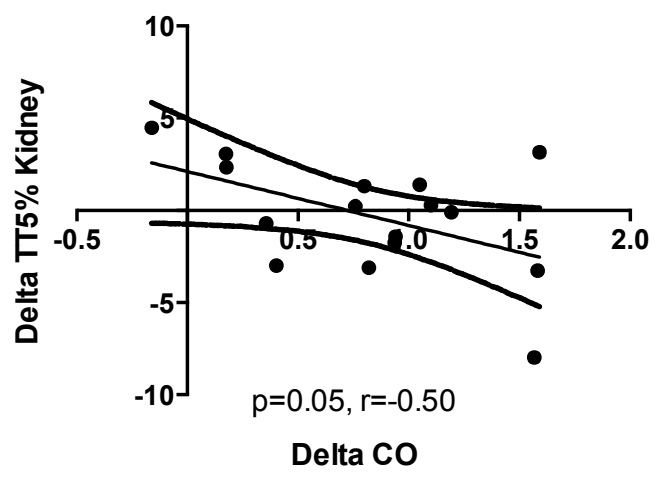

Figure 6 